\title{
The gravitoelectric nuclear energy
}

\author{
Roberto Napolitano* \\ ORCID: https://orcid.org/0000-0002-0985-1982 \\ *e-mail: lennyus@ alice.it
}

Keywords: gravitoelectrostatic force; gravitoelectric energy; nuclear structure; nuclear force

\begin{abstract}
Newtonian mechanics has been abandoned, perhaps mistakenly, in the study of dynamics at microscopic scales, in particular at subatomic scales, in favor of quantum mechanics, relied on a probabilistic and indeterministic way of conceiving the reality at those scales.

Though, by means of Newtonian mechanics, we show that, at subatomic level, Nature behaves similarly to the macroscopic level, being possible to find traces of the existence of gravity even within the atomic nucleus, where, more precisely, the gravitoelectrostatic force acts as a centripetal force, having this magnitude $F_{g e s}=\frac{G M^{2}}{\pi^{2} \varepsilon_{0} R^{4}}$ (eq. 18), which is $10^{38}$ times stronger than the force of gravity at same range, where $M$ is the mass of the nucleus, $R$ is twice the nuclear radius observed in the electron scattering experiments and $\varepsilon_{0}$ is the dielectric constant in vacuum.

By supposing that the gravitoelectric potential energy, analogously to the gravitational potential energy, be expressed as $U_{g e}=-F_{g e s} * R$, we obtain surprising outcomes.

The new approach offers an occasion for discussing not only about the preferable method to follow in physics - which turns out to be the induction, rather than the deduction - but also about the physics foundations, in particular about the nature of the atomic nucleus, which perhaps should be reconsidered in deterministic terms, rather than probabilistic ones.

The "Third rule of reasoning in philosophy" contained in Newton's Philosophiae Naturalis Principia Mathematica is applied, in particular the analogy among natural phenomena is used as a crucial tool for discovering fundamental laws of Nature.

A new theoretical form of eco-sustainable exploitation of nuclear energy is envisaged.
\end{abstract}

\section{Introduction}

The world of subatomic particles is weird, difficult to describe according to the knowledge achieved by classical physics, based on the observation of macroscopic phenomena, showing a deterministic and predictable behaviour.

Probably the experiment that more than others has opened a new view on this strange world has been the two slits experiment, which demonstrated that a photon (but the experiment has 
been confirmed even for electrons) passes simultaneously through two slits in a plate, and this is considered the evidence of the dual nature of subatomic particles, both of wave and of particle.

Even though the ubiquity of subatomic particles due to the their wave nature is well-described by quantum mechanics, anyway this is only a partial description of their behaviour, because of their dual nature, undulatory and corpuscular simultaneously, and in this study we will seek to demonstrate that a deterministic description of the nucleonic behaviour, as particles, is however possible, in particular (and surprisingly) by means of Newtonian mechanics.

In detail we propose a new approach, completely classic, to the study of the atomic nucleus, through the explanation of its size.

\section{Experimental evidences of nuclear size}

The force acting within the atomic nucleus as responsible for the nuclear stability and for the cohesion of nucleons is, according to the nuclear standard physics, the strong nuclear force.

Though, there are some evidences that seem to deny the existence of this force.

First of all, if the strong nuclear force were responsible for the cohesion of nucleons, it should explain the nuclear size, which still remains without a theoretical motivation instead.

In particular, in the electron scattering experiments it has been demonstrated [1] that the nuclear radius, for medium and heavy atoms, follows this empirical law:

$$
R=1.21 * \sqrt[3]{A} \mathrm{fm}
$$

where $A$ is the mass number, namely the sum of protons and neutrons present in the nucleus considered.

\section{Results}

In this work we will demonstrate that the above empirical formula can receive a theoretical explanation in the framework of our approach, in particular by applying eq. (14) one can derive the following relation for the nuclear radius $R$ :

$$
R=\sqrt[3]{\frac{4 G K M}{\pi c^{2}}} \mathrm{fm}
$$

where 1 femtometer (fm) equals $10^{-15}$ meter.

A consequential finding is the discovery of the natural law contained in eq. (2):

$$
\left\langle E_{K}\right\rangle=-\frac{1}{2} *\langle\phi\rangle
$$


where $\langle\phi\rangle$ is the average over time of the potential energy of the orbiting body subjected to the gravitational or to the gravitoelectrostatic force, and $\left\langle E_{K}\right\rangle$ is the average over time of its kinetic energy.

The above law is inferable by induction from the comparison of the following three equations:

a) As concerns the planetary orbits, we show in eq. (1d) that $\frac{G M m}{R}=m v^{2}$, where $M$ is the Solar mass, $m$ is the mass of whatever planet of the Solar System, $R$ is the semi-major axis of the planetary orbit and $v$ is its average orbital velocity;

b) As regards the nucleonic orbit, we show in eq. (14) that $\frac{4 G K M^{2}}{\pi R^{3}}=M c^{2}$, where $M$ is the mass of the atomic nucleus considered, $R$ is twice the nuclear radius detected in the electron scattering experiments, and $c$ is the speed of light;

c) As concerns the black hole, we show in eq. (17) that $\frac{4 G M^{2}}{R}=M c^{2}$, where $M$ is the mass of the black hole considered and $R$ is the radius of the sphere within which the mass of the black hole is confined.

$G$ is the gravitational constant and $K$ is the Coulomb constant.

\section{Discussion}

The inexistence of the strong nuclear force, inferred from an impartial analysis of what happens in the alpha decay of U238, seems to have been confirmed in 2009 by an experiment, reported in the reference [2], because, while the range of maximum actuation of the strong force is less than $3 \mathrm{fm}$, the experiment has detected that a halo neutron in Be11 is separated from the rest of the nucleus by a distance of $7 \mathrm{fm}$.

In consideration of this discrepancy between the prevailing nuclear theoretical model and the aforesaid experiment, in the present work we will seek to provide evidences of the existence of gravity even within the atomic nucleus, as the force responsible for the nuclear stability and for the cohesion of nucleons.

From Einstein's theory of relativity we know that the energy contained in the atomic nucleus is equal to $E=M c^{2}$, where $M$ is the mass of the nucleus and $c$ is the speed of light in vacuum.

We will try to demonstrate that the above formula is incomplete, and that, once completed with the missing part, it will appear entirely nude to us, without that halo of mystery that previously prevented us from seeing more deeply, showing that perhaps it does not denote the mass-energy equivalence principle proposed by Einstein's special relativity, but something else. 
By the force of gravity being conservative, then the gravitational potential energy $U$ held by a mass body $m$ subjected to gravitational force exerted by a mass body $M$, is expressed by the following relation:

$$
U=-F_{g} * R
$$

where $F_{g}$ is the force of gravity $\frac{G M m}{R^{2}}$ and $R$ is the distance between the centers of the two bodies (assumed as spherical).

Therefore eq. (1) becomes:

$$
\begin{aligned}
& U=-\frac{G M m}{R^{2}} * R \\
& U=-\frac{G M m}{R}
\end{aligned}
$$

The above relation expresses the gravitational potential energy of the mass body $m$, which is usually defined as the test body.

Eq. (1a) takes on particular importance in the contest of the virial theorem.

Let's see why.

The virial theorem (by R. Clausius 1870), for a central potential $\langle\phi\rangle(\vec{R})=\phi(R) \propto \pm R^{ \pm b}$, states that:

$$
\left\langle E_{K}\right\rangle= \pm \frac{b}{2} *\langle\phi\rangle
$$

where $\langle\phi\rangle$ is the average over time of the potential energy of the test body, $\left\langle E_{K}\right\rangle$ is the average over time of its kinetic energy and $b$ is the exponent of the distance $R$ as it appears in the formula of the potential energy.

As the gravitational potential energy $U$ is inversely proportional to the distance $R$ [see eq. (1a)], then $b$ is equal to -1 :

$$
\left\langle E_{K}\right\rangle=-\frac{1}{2} *\langle U\rangle
$$

So, applying eq. (1c) to the Solar System, and considering $M$ as the mass of the Sun, $m$ as the mass of the planet under consideration, $R$ as the mean distance planet-Sun, namely the semimajor axis of the planetary orbit, and $v$ as the mean orbital velocity of the planet, we have:

$$
\begin{gathered}
\frac{1}{2} m v^{2}=-\frac{1}{2} *\left(-\frac{G M m}{R}\right) \\
\frac{1}{2} m v^{2}=\frac{1}{2} * \frac{G M m}{R} \\
\frac{G M m}{R}=m v^{2}
\end{gathered}
$$

Let's test now eq. (1d) on Venus planet, which shows the least eccentric orbit among all the planets of the Solar System, namely an orbit which is well-approximable to a circumference, and 
let's replace the numeric value of the mass, of the mean orbital velocity and of the semi-major axis of Venus in eq. (1d).

By knowing that:

the mass $m$ of Venus is $4.8675 * 10^{24} \mathrm{~kg}$

the mass $M$ of the Sun is $1.9891 * 10^{30} \mathrm{~kg}$

the average orbital velocity $v$ of Venus is $35,020 \mathrm{~m} / \mathrm{s}$

the semi-major axis $R$ of the Venus' orbit is $108,210,123,792 \mathrm{~m}$

the gravitational constant $G$ is equal to $6.6743 * 10^{-11} \mathrm{~N} * \mathrm{~m}^{2} * \mathrm{~kg}^{-2}$

then eq. (1d) leads to:

$$
\begin{gathered}
5.9717 * 10^{33} \text { joule }=5.9695 * 10^{33} \text { joule } \\
-\frac{U}{m v^{2}}=\frac{5.9717 * 10^{33}}{5.9695 * 10^{33}}=1.0003
\end{gathered}
$$

Eq. (1d) holds and consequently eq. (1b) is true, or, better saying, seems to be true.

In fact we will demonstrate that the virial theorem expressed in eq. (1b) is incorrect, whereas the correct law would be the following:

$$
\left\langle E_{K}\right\rangle=-\frac{1}{2} *\langle\phi\rangle
$$

The difference is remarkable, because the right-hand side of eq. (1b) depends on the exponent $b$ of the distance $R$, whereas the right-hand side of eq. (2) is independent from the aforesaid exponent.

In particular in this study we will demonstrate that in Nature there exists at least one case in which the gravitational potential energy is inversely proportional to the third power of the distance $\left(\frac{1}{R^{3}}\right)$ and in this case we will show that the virial theorem [eq. (1b)] does not hold, whereas eq. (2) perfectly holds.

We are referring to the atomic nucleus, within which we will show that the force of gravity and the electrostatic force merge, giving rise to a force that we will define gravitoelectrostatic force $\left(F_{g e s}\right)$, having this magnitude:

$$
F_{g e s}=\frac{G K M^{2}}{2 \pi R^{4}}
$$

where $K$ is the Coulomb's constant, $G$ is the gravitational constant, $M$ is the mass of the nucleus and $R$ is the nuclear radius (for now we leave out the dimensional analysis, addressed in chapter 4.2).

It's paramount to specify that we have found this value inductively, namely by attempts, not by means of any deductive, mathematical derivation, according to the Newton's idea that physics has to be intended as an experimental philosophy, in which induction has a preeminent role in discovering the laws of Nature (for further insights, see chapter 4.4). 
It's also important to underline that eq. (3) is only one of the two possible forms that the gravitoelectrostatic force can assume, which can also be expressed in the similar form contained in eq. (12), like we will see in chapter 4.4.

By supposing that the gravitoelectrostatic force be conservative and that the gravitoelectric potential energy be negative, analogously to the gravitational force and energy, respectively, then eq. (1) becomes:

$$
\begin{gathered}
U_{g e}=-F_{g e s} * R \\
U_{g e}=-\frac{G K M^{2}}{2 \pi R^{4}} * R \\
U_{g e}=-\frac{G K M^{2}}{2 \pi R^{3}}
\end{gathered}
$$

where $U_{g e}$ stands for the gravitoelectric potential energy, in order to distinguish it from the gravitational potential energy, which is usually indicated with the letter $U$.

Now, in order to mathematically demonstrate that the equations (2) and (5) are true, we have to verify if eq. (2) holds even by taking, as $\langle\phi\rangle$, the right-hand side of eq. (5), and, as $\left\langle E_{K}\right\rangle$, the kinetic energy of nucleons, which are assumed to be self-orbiting particles, namely staying at rest and, at same time, revolving at the speed of light around themselves thanks to the gravitoelectrostatic force acting as a centripetal force, consequently their kinetic energy can be considered as constant and equal to $\frac{1}{2} M c^{2}$, then eq. (2) becomes:

$$
\begin{gathered}
\frac{1}{2} M c^{2}=-\frac{1}{2}\left(-\frac{G K M^{2}}{2 \pi R^{3}}\right) \\
\frac{1}{2} M c^{2}=\frac{1}{2} * \frac{G K M^{2}}{2 \pi R^{3}} \\
\frac{G K M^{2}}{2 \pi R^{3}}=M c^{2}
\end{gathered}
$$

where $R$ is the nuclear radius detected in the electron scattering experiments: for medium and heavy atoms, $R=1.21 * \sqrt[3]{A} \mathrm{fm}$, where $A$ is the mass number (see reference [1]).

It's important to specify that the mass of the nucleus, $M$, has been taken as the sum of the masses of protons and of neutrons, without taking into account the binding energy (mass-defect), that therefore has not been subtracted from the mentioned sum.

Let's test now eq. (6), considering the nucleus of bromum atom $\left({ }^{79} \mathrm{Br}\right)$, which contains 35 protons and 44 neutrons, whose radius - according to the empirical formula $R=1.21151 *$ $\sqrt[3]{A} \mathrm{fm}-$ is $5.1983 \mathrm{fm}:$

$$
\frac{\left(6.6743 * 10^{-11}\right) *\left(8.9875 * 10^{9}\right) *\left\{[(35 * 1.6726)+(44 * 1.6749)] * 10^{-27}\right\}^{2}}{2 * 3.1415 *\left(5.1983 * 10^{-15}\right)^{3}}=[(35 * 1.6726)+(44 * 1.6749)] * 10^{-27} * c^{2}
$$

where $c$ is the speed of light in vacuum: $299,792,458 \mathrm{~m} / \mathrm{s}$ 


$$
\begin{gathered}
1.1884 * 10^{-8} \frac{\mathrm{N}^{2}}{\mathrm{C}^{2}} * \mathrm{~m}=1.1884 * 10^{-8} \text { joule } \\
-\frac{U_{g e}}{M c^{2}}=\frac{1.1884 * 10^{-8}}{1.1884 * 10^{-8}}=1
\end{gathered}
$$

The above equivalence holds under the assumption that $\frac{\mathrm{N}^{2}}{\mathrm{C}^{2}} * \mathrm{~m} \equiv$ joule, and consequently that $\frac{\mathrm{N}^{2}}{\mathrm{C}^{2}} \equiv \mathrm{N}$, and we will address this issue in chapter 4.2 .

For summary reasons it's not worth reporting here the above calculation for all the atoms, since the empirical formula of the nuclear radius seen above $(R=1.21151 * \sqrt[3]{A} \mathrm{fm})$ is applicable to every medium and heavy atom.

The only further atom that we can consider as a demonstration of the validity of eq. (6) is the lead atom, the heaviest among the stable atoms.

The nucleus of lead atom contains 82 protons and 126 neutrons, and its radius, according to the mentioned empirical formula $R=1.21151 * \sqrt[3]{A} \mathrm{fm}$, is $7.1781 \mathrm{fm}$, hence, applying eq. (6), we obtain the following values:

$$
\begin{gathered}
3.1295 * 10^{-8} \frac{\mathrm{N}^{2}}{\mathrm{C}^{2}} * \mathrm{~m}=3.1293 * 10^{-8} \text { joule } \\
-\frac{U_{g e}}{M c^{2}}=\frac{3.1295 * 10^{-8}}{3.1293 * 10^{-8}}=1.00005
\end{gathered}
$$

In order to support our claim that the force contained in equation (3) acts as a centripetal force within the nucleus, analogously to the gravitational force exerted by the Sun upon the planets of the Solar System, it suffices to divide both sides of eq. (6) by the nuclear radius $R$ :

$$
\frac{G K M^{2}}{2 \pi R^{4}}=\frac{M c^{2}}{R}
$$

The right-hand side of the above equation denotes the centripetal force to which nucleons are subjected during their revolution around the center of nucleus, and this confirms that the gravitoelectrostatic force, expressed by the left-hand side of the mentioned equation, acts as a centripetal force, analogously to the solar gravitational force experienced by the planets.

We reiterate that $M$, which appears in formulae (5) and (6), is taken as the total mass of nucleons, intended as the sum of the masses of protons and of neutrons, without taking into account the mass-defect detected in the nuclear reaction experiments and ascribed, by the nuclear standard physics, to the binding energy of nucleons, which therefore has not been subtracted from the mentioned sum of the nucleonic masses, and, despite this, equation (6) perfectly holds, and this seems to demonstrate that the mass-defect detected in the nuclear reactions is not the consequence of the mass-energy equivalence principle stated by Einstein's special theory of relativity, but is most likely ascribable to the increasing of nuclear radius. 
In other terms, from the relevant mathematical findings achieved in eq. (6), it seems possible to infer that the mass of an unbound nucleon (proton or neutron) is not greater than that of a bound nucleon, but is exactly the same, and that the discrepancy detected in nuclear reactions is not due to the mass defect of a bound nucleon with respect to a free nucleon, but is the consequence of the very probable increasing of nuclear radius occurring in the nuclear reactions which in turn implies, according to eq. (5), the decrease of nuclear potential energy (assuming that the velocity of nucleons, $c$, remains constant), which is very likely responsible for what is detected in nuclear reactions and interpreted, perhaps mistakenly, as the mass-defect by the nuclear standard physics.

\subsection{Is the virial theorem always valid?}

As we have already expressed in eq. (1b), reported below, the virial theorem states, for a central potential $\langle\phi\rangle(\vec{R})=\phi(R) \propto \pm R^{ \pm b}$, that:

$$
\left\langle E_{K}\right\rangle= \pm \frac{b}{2} *\langle\phi\rangle
$$

where $\langle\phi\rangle$ is the average over time of the potential energy, $\left\langle E_{K}\right\rangle$ is the average over time of the kinetic energy and $b$ is the exponent of the distance $R$ as it appears in the formula of the potential energy.

Since the gravitational potential energy $U$ is inversely proportional to the distance $\left(U=-\frac{G M m}{R}\right)$, then the exponent $b$ of the distance is equal to -1 and eq. (1b) becomes:

$$
\left\langle E_{K}\right\rangle=-\frac{1}{2} *\langle U\rangle
$$

which is true for the gravitational systems, yet, in the light of the findings reached in eq. (6), whose left-hand side denotes quite indisputably the gravitoelectric nuclear potential energy, eq. (1b) does not hold anymore.

In fact, by the gravitoelectric nuclear potential energy being $U_{g e}=-\frac{G K M^{2}}{2 \pi R^{3}}$, then the exponent $b$ of the distance $R$ that should appear in eq. (1b) would be -3 , so that the necessary condition to have a stable nucleonic orbit, according to the virial theorem and taking the constant $c$ as the velocity of nucleons, would turn out to be:

$$
\begin{gathered}
\frac{1}{2} M c^{2}=-\frac{3}{2} *\left(-\frac{G K M^{2}}{2 \pi R^{3}}\right) \\
\frac{1}{2} M c^{2}=\frac{3}{2} * \frac{G K M^{2}}{2 \pi R^{3}}
\end{gathered}
$$

Multiplying both sides by 2 :

$$
M c^{2}=\frac{3 G K M^{2}}{2 \pi R^{3}}
$$


which is not true.

In fact, if we apply the above equation again to the bromum atom $\mathrm{Br} 79$, it leads to:

$$
\frac{M c^{2}}{\left(\frac{3 G K M^{2}}{2 \pi R^{3}}\right)}=\frac{1.1884 * 10^{-8}}{3.5652 * 10^{-8}} \neq 1
$$

The virial theorem, therefore, does not hold in the atomic nucleus.

This happens because it is implicitly based on a premise (namely the fact that the force of gravity cannot be other than inversely proportional to the square of the distance, and consequently that the gravitational potential energy cannot be other than proportional to $R^{-1}$ ) which is not logically demonstrable, and this implies that it cannot be considered a theorem in the proper sense of the term, because a theorem is, by definition, a proposition which can be scientifically demonstrated, and this obviously holds even for its logical premises.

Therefore one should admit that eq. (1b) should be replaced by eq. (2), reported below:

$$
\left\langle E_{K}\right\rangle=-\frac{1}{2} *\langle\phi\rangle
$$

The above equation applies to every form of potential energy (both gravitational and gravitoelectric), regardless of its mathematical configuration, because it does not depend on the exponent of the distance appearing in the formula of the potential energy, consequently it holds even in the atomic nucleus, where we have seen that the potential energy of nucleons is proportional to $R^{-3}$ [see eq. (6) and relative demonstration].

In other words, in this scenario one should admit that eq. (2) be a fundamental principle of Nature existing in every gravitational system, in the sense that it would not have any mathematical derivation, but should be accepted as it is.

After all, there are some aspects of the force of gravity that are not entirely explainable, just think of the fact, we repeat, that it depends, without any apparent logical reason, on the inverse of the square of the distance.

However the aim of this paper is not getting into the details of the debate between those who believe in the existence of the fundamental laws of Nature, and those who believe that the physical laws are created by humans to describe the reality and consequently that every natural law should be explainable in the light of the reason, but it's undeniable that the answer to the question here proposed depends on the way of solving this dispute.

The only thing that we can say in this regard is that the deductive method doesn't seem the best way of approaching the force of gravity, as it is shown by the paradoxical results of the virial theorem seen above.

The inductive method, on the contrary, by starting from single cases in order to infer the existence of a general principle [in our case, that expressed by eq. (2)], seems to be more suitable 
to study the issues related to gravity and electricity, which, as for every phenomenological entity, are not a-priori knowable in their every single aspect.

For clarity, we report below equations (6) and (1d), in order to show their strong resemblance:

- As regards the nucleonic orbit [eq. (6)]: $\frac{G K M M}{2 \pi R^{3}}=M c^{2}$

- As concerns the planetary orbit [eq. (1d)]: $\frac{G M m}{R}=m v^{2}$

These two equations are evidently two specific applications of the general principle contained in eq. (2), which for clarity is reported below multiplying both sides by 2 :

$$
-\langle\phi\rangle=2 *\left\langle E_{K}\right\rangle
$$

The inductive method characterizes all branches of natural science (as biology, chemistry, medicine and so on), and there are no reasons for physics to represent an exception to this modus operandi, whereas there are obvious reasons for which only mathematics and geometry which do not fall within the natural sciences but are the product of the human intellect - be subjected to the deductive method.

But Nature does not care about our sense of logic, our sense of mathematical elegance: it behaves according to its sense of beauty, that could be different from ours.

After all, those, like Einstein and many other physicists, who attempted or attempt to treat the force of gravity as an abstract geometrical entity, by finding its logical primary cause in the spacetime, in particular in its curvature, inevitably fall into contradiction, by postulating that the magnitude of this force is not explainable in the light of reason, accepting it as it is, namely implicitly supposing that it cannot be other than inversely proportional to the square of the distance, without any geometrical, logical, mathematical, scientific explanation justifying this point too.

\subsection{The gravitoelectrostatic force seen in the light of the dimensional analysis: the gravitoelectric zone}

According to the dimensional analysis, the gravitoelectrostatic force, that in eq. (3) we have supposed to be existing in the atomic nucleus, scales as the square of a force over a charge squared or, in units of the International System, as $\left(\frac{\mathrm{N}}{\mathrm{C}}\right)^{2}$, therefore it does not seem to be a force, which should be measured in newton $(\mathrm{N})$, and the gravitoelectric energy would be measured in $\left(\frac{\mathrm{N}}{\mathrm{C}}\right)^{2} * \mathrm{~m}$ [see eq. (3a)], which does not seem to be an energy, usually measured in joule $(\mathrm{N} * \mathrm{~m})$ or in electronvolt.

This is an obvious problem that arises every time a new force is discovered, but is solvable, like we will see shortly. 
In fact we know that $\frac{\mathrm{N}}{\mathrm{C}}$ is the derived unit of measurement for the electric field, that is considered as a force, in particular it is traditionally defined [3 - 5] as a vector that associates to each point in space the (electrostatic) force per unit of charge exerted on an infinitesimal positive test charge at rest at that point.

By using the above definition, we can define the nuclear gravitoelectric zone (intended only in spatial sense, and defined like this in order to distinguish it from the Einsteinian space-temporal gravitational field), as a vector that associates to each point in space the gravitoelectrostatic force per unit charge exerted by the nucleus upon itself (and this justifies the square of the unit $\frac{\mathrm{N}}{\mathrm{C}}$ ) considered at rest at that point.

From the fact that the nucleus is a self-interacting system, hence, it follows that the gravitoelectrostatic force and the gravitoelectric zone coincide, because the gravitoelectric zone is not placed outside the nucleus, but is within it, and for this reason we will indicate them with the same acronym $F_{g e s}$.

In fact in this regards it is worth to remark that, as concerns the electric field $(E)$, the electrostatic force differs from the mentioned field because this latter is given by the ratio between the electrostatic force and the test charge $\left(E=\frac{k Q q}{R^{2}} * \frac{1}{q}=\frac{k Q}{R^{2}}\right)$, but this is not possible in the nuclear gravitoelectric zone, because the gravitoelectrostatic force $\left(F_{g e s}=\frac{G K M^{2}}{2 \pi R^{4}}\right)$ cannot be divided by the test mass $(m)$, since this latter lacks, by the nucleus being a selfinteracting system $(M * M)$, and consequently the gravitoelectric zone must have the same value as the gravitoelectrostatic force.

Hence, from the numerical identity seen in (6a) we can infer, by induction, the existence of a new force that we will define gravitoelectrostatic force, measured in $\left(\frac{\mathrm{N}}{\mathrm{C}}\right)^{2}$.

The square of $\frac{N}{C}$ is justified, we repeat, by the fact that the gravitoelectrostatic force acts upon the same body that produces it, in essence, is a self-interacting force.

Consequently the gravitoelectric potential energy $U_{g e}$ will be measured in $\left(\frac{\mathrm{N}}{\mathrm{C}}\right)^{2} * \mathrm{~m}$ [see eq. (3a)], which, from the numerical identity shown in eq. (6a), must be equivalent to 1 joule:

$$
1 \mathrm{~J} \equiv\left(\frac{\mathrm{N}}{\mathrm{C}}\right)^{2} * \mathrm{~m}
$$

We remark that the equivalence $1 \mathrm{~J} \equiv\left(\frac{\mathrm{N}}{\mathrm{C}}\right)^{2} * \mathrm{~m}$ is crucial to demonstrate the validity of our theory, since the derived unit $\left(\frac{\mathrm{N}}{\mathrm{C}}\right)^{2} * \mathrm{~m}$, in turn equivalent to $\frac{\mathrm{V}^{2}}{\mathrm{~m}}$, does not need to be converted in the corresponding value of 1 joule, by being exactly equal to it, differently from the unit of 
measurement generally used for energy at microscopic scale, namely the electronvolt (eV), which has a different value from 1 joule $\left(1 \mathrm{eV}=1.60217^{-19} \mathrm{~J}\right)$.

But from eq. (9) another important aspect does emerge.

Since $1 \mathrm{~J}$ is equivalent to $\mathrm{N} * \mathrm{~m}$, then eq. (9) becomes:

$$
\begin{aligned}
\mathrm{N} * \mathrm{~m} & \equiv\left(\frac{\mathrm{N}}{\mathrm{C}}\right)^{2} * \mathrm{~m} \\
\mathrm{~N} & \equiv \frac{\mathrm{N}^{2}}{\mathrm{C}^{2}}
\end{aligned}
$$

This is possible only if we admit that:

$$
1 \mathrm{C} \equiv \sqrt{1 \mathrm{~N}}
$$

In fact, under this assumption, eq. (10) would become:

$$
\begin{gathered}
\mathrm{N}=\frac{\mathrm{N}^{2}}{(\sqrt{\mathrm{N}})^{2}} \\
\mathrm{~N}=\frac{\mathrm{N}^{2}}{\mathrm{~N}} \\
\mathrm{~N}=\mathrm{N}
\end{gathered}
$$

But how is it possible that $\mathrm{N} \equiv \mathrm{C}^{2}$

How can the square of the charge's unit of measurement be equivalent to the unit of measurement of force?

In this regards it's worth to say that this is not so weird, because this situation is very similar to what it happens in CGS (centimeter-gram-second) system of units.

In the mentioned system, in fact, charge is measured in two alternative ways:

a) In the electrostatic units variant of the CGS system (CGS-ESU), charge is measured in statcoulomb or esu charge (also known as franklin, Fr), which is equivalent to $\sqrt{1 \mathrm{dyn}} * 1 \mathrm{~cm} \equiv 1 \mathrm{~g}^{\frac{1}{2}} * 1 \mathrm{~cm}^{\frac{3}{2}} * 1 \mathrm{~s}^{-1}$, where dyn stands for the dyne (equivalent to $1 \mathrm{~g} * 1 \mathrm{~cm} * 1 \mathrm{~s}^{-2}$ ), the unit of measurement of force in the CGS-ESU system. This system is defined coherent and "absolute", because the dimensions of the electrical quantities are expressible in terms of units of the mechanical quantities, i.e. mass, length and time.

b) In the electromagnetic units variant of the CGS system (CGS-EMU), charge is measured in $\mathrm{Bi} * \mathrm{~s}$, where $\mathrm{Bi} \equiv \sqrt{1 \mathrm{dyn}}$ and stands for the biot (the EMU unit of current), also known as abcoulomb or emu charge, therefore in CGS-EMU system charge is measured in $\sqrt{1 \mathrm{dyn}} * 1 \mathrm{~s} \equiv 1 \mathrm{~g}^{\frac{1}{2}} * 1 \mathrm{~cm}^{\frac{1}{2}}$

As we notice, in both the variants of CGS system, the square root of the unit of force (dyne) is involved in the unit of measurement of charge (in the first case multiplied by the centimeter, $\mathrm{cm}$, 
in the second multiplied by the second, s), and this strongly supports the correctness of our result, according to which, in the International System of Units (SI), the unit of measurement of charge, the coulomb, turns out to be equivalent to the square root of the unit of force, the newton, and in this way the mentioned system becomes coherent without the need of introducing a specific unit of measurement for the electrical quantities (that is the ampere), being possible to express these latter in terms of the three (fundamental) quantities used in mechanics for the motion of bodies - namely mass, length and time - thus becoming an "absolute" system of units, analogously to the CGS-ESU system:

$$
\begin{gathered}
1 \text { coulomb } \equiv \sqrt{1 \text { newton }} \\
1 \mathrm{C} \equiv \sqrt{\frac{1 \mathrm{Kg} * 1 \mathrm{~m}}{1 \mathrm{~s}^{2}}} \\
1 \mathrm{C} \equiv \frac{1}{\mathrm{~s}} \sqrt{1 \mathrm{Kg} * 1 \mathrm{~m}} \\
1 \mathrm{C} \equiv 1 \mathrm{~kg}^{\frac{1}{2}} * 1 \mathrm{~m}^{\frac{1}{2}} * 1 \mathrm{~s}^{-1}
\end{gathered}
$$

Consequently the Coulomb constant $K$ would be expressed in $\mathrm{m}^{2}$ in the SI:

$$
\mathrm{K}=8.9875 * 10^{9} \frac{\mathrm{N} * \mathrm{~m}^{2}}{\mathrm{C}^{2}}
$$

By being $\mathrm{C}^{2} \equiv \mathrm{N}$, then

$$
\mathrm{K}=8.9875 * 10^{9} \mathrm{~m}^{2}
$$

And the electrostatic force $F_{e}$ would be nonetheless measured in newton:

$$
\begin{gathered}
\mathrm{F}_{e}=\frac{K * Q q}{d^{2}} \\
\mathrm{~F}_{e}=\frac{\mathrm{m}^{2} * \mathrm{C}^{2}}{\mathrm{~m}^{2}} \\
\mathrm{~F}_{e}=\frac{\mathrm{m}^{2} * \mathrm{~N}}{\mathrm{~m}^{2}} \\
\mathrm{~F}_{e}=\mathrm{N}
\end{gathered}
$$

This is the only way to avoid to assert, quite unrealistically, that the numerical identity seen in eq. (6a) is only a fortuitous coincidence.

From eq. (6), reported below, we can show that the units of measurement of nuclear mass, size and volume are perfectly coherent with the SI, in fact they are, respectively, $\mathrm{Kg}, \mathrm{m}$ and $\mathrm{m}^{3}$.

As regards the mass:

$$
\begin{gathered}
\frac{G K M^{2}}{2 \pi R^{3}}=M c^{2} \\
M=\frac{2 \pi R^{3} c^{2}}{G K} \\
\mathrm{Kg}=\frac{\mathrm{m}^{3} * \frac{\mathrm{m}^{2}}{\mathrm{~s}^{2}}}{\left(\frac{\mathrm{N} * \mathrm{~m}^{2}}{\mathrm{~kg}^{2}} * \frac{\mathrm{N} * \mathrm{~m}^{2}}{\mathrm{C}^{2}}\right)}
\end{gathered}
$$




$$
\mathrm{Kg}=\frac{\frac{\mathrm{m}^{5}}{\mathrm{~s}^{2}}}{\left(\frac{\mathrm{N}^{2}}{\mathrm{C}^{2}} * \frac{\mathrm{m}^{4}}{\mathrm{Kg}^{2}}\right)}
$$

By we having demonstrated in eq. (10) that $\frac{\mathrm{N}^{2}}{\mathrm{C}^{2}}$ is equivalent to $\mathrm{N}$, then:

$$
\begin{gathered}
\mathrm{Kg}=\frac{\frac{\mathrm{m}^{5}}{\mathrm{~s}^{2}}}{\left(\frac{\mathrm{N} * \mathrm{~m}^{4}}{\mathrm{Kg}^{2}}\right)} \\
\mathrm{Kg}=\frac{\mathrm{Kg}^{2} * \mathrm{~m}}{\mathrm{~N} * \mathrm{~s}^{2}} \\
\mathrm{Kg}=\frac{\mathrm{Kg} * \mathrm{~m}}{\mathrm{~s}^{2}} * \frac{\mathrm{Kg}}{\mathrm{N}}
\end{gathered}
$$

By knowing that $\frac{\mathrm{Kg} * \mathrm{~m}}{\mathrm{~s}^{2}}$ is equivalent to $\mathrm{N}$, then:

$$
\begin{gathered}
\mathrm{Kg}=\frac{\mathrm{N} * \mathrm{Kg}}{\mathrm{N}} \\
\mathrm{Kg}=\mathrm{Kg}
\end{gathered}
$$

As regards the size of the nucleus:

$$
\begin{gathered}
\frac{G K M^{2}}{2 \pi R^{3}}=M c^{2} \\
R=\sqrt[3]{\frac{G K M}{2 \pi c^{2}}} \\
\mathrm{~m}=\sqrt[3]{\frac{\frac{{\mathrm{N} * \mathrm{~m}^{2}}_{\mathrm{kg}^{2}} * \frac{\mathrm{N} * \mathrm{~m}^{2}}{\mathrm{C}^{2}} * \mathrm{Kg}}{\frac{\mathrm{m}^{2}}{\mathrm{~s}^{2}}}}{\mathrm{~m}=\sqrt[3]{\frac{\mathrm{N}^{2}}{\mathrm{C}^{2}} * \frac{\mathrm{m}^{2}}{\mathrm{Kg}} * \mathrm{~s}^{2}}}}
\end{gathered}
$$

By we having demonstrate in eq. (10), we repeat, that $\frac{\mathrm{N}^{2}}{\mathrm{C}^{2}} \equiv \mathrm{N}$, then:

$$
\begin{gathered}
\mathrm{m}=\sqrt[3]{\mathrm{N} * \frac{\mathrm{m}^{2} * \mathrm{~s}^{2}}{\mathrm{Kg}}} \\
\mathrm{m}=\sqrt[3]{\frac{\mathrm{kg} * \mathrm{~m}}{\mathrm{~s}^{2}} * \frac{\mathrm{m}^{2} * \mathrm{~s}^{2}}{\mathrm{Kg}}} \\
\mathrm{m}=\sqrt[3]{\mathrm{m}^{3}} \\
\mathrm{~m}=\mathrm{m}
\end{gathered}
$$

Consequently the unit of measurement of the volume occupied by the atomic nucleus is $\mathrm{m}^{3}$.

It's important to remark that the fact that the nuclear gravitoelectrostatic force depends only on the square of the nuclear mass, not also on the square of the charge of protons, is not so weird, because the mass is the common element among nucleons (both protons and neutrons have a mass, but only the firsts have a charge). 
Anyway, since the nucleus is overall charged, although contains also neutrons, then the presence of the Coulomb constant along with the gravitational constant is logically explainable, despite the force does not depend on the square of the charges.

Moreover it's worth to remark that the equation of nuclear mass derivable from eq. (6), $M=\frac{2 \pi R^{3} c^{2}}{G K}$, does not give rise to the problems of infinites which are inherent in Maxwell's equations.

We know in fact that, in Maxwell's equations, the total mass $m_{t}$ of a charged particle is given by the sum of the rest mass $m_{0}$ with the energy of the electrostatic field generated by the charged particle itself:

$$
m_{t}=m_{0}+\frac{q^{2}}{8 \pi r_{e}}
$$

where $r_{e}$ is the radius of the particle.

From the above formula it emerges all the paradoxicalness of the Maxwell's equation, since the mass of the particle would enhance if its radius decreased, so that, for an extremely small particle, the mass would become infinite, and this issue is "solved" by the traditional physics by resorting to the renormalization technique, whereas, from our equation of mass, $M=\frac{2 \pi R^{3} c^{2}}{G K}$, there emerges no paradoxical numerical situation, and consequently there is no need to use the questionable renormalization technique at all.

The numerical identity seen in eq. (6a) is so perfect that an underlying physical meaning must subsist beyond any reasonable doubt, by being quite unconceivable to simply assert that one deals with a mere accident.

But what is this underlying physical meaning of eq. (6)?

In the next chapter we'll try to discuss this issue.

\subsection{Are nucleons self-orbiting particles?}

The result achieved above gives rise to a philosophical question.

How to interpret eq. (5)?

In other words, the fact that the gravitoelectric potential energy expressed by eq. (5) depends on the mass of nucleons squared, could have a precise physical meaning, in particular could mean that the nucleons stay both in the center of the nucleus and, at same time, in orbit around it, because we have replaced, in the equation (3) regarding the gravitoelectrostatic force, the mass $m$ — which denotes the orbiting body, having a very small mass with respect to the central one with the mass $M$, that is the total mass of nucleons. 
If we accept this assumption, there would be non-irrelevant consequences on the foundations of physics, because this would mean that the nucleons would not be everywhere, like instead the indeterministic vision of quantum mechanics proposes, but would be only in two specific points and conditions simultaneously (at rest and revolving), always having, even before the measurement, a precise position, trajectory and velocity in while they are orbiting about the center of the nucleus (occupied by their alter ego at rest).

In this weird scenario, one should accept not only the idea that the nucleons stay in two places at the same time, but also the fact that they are both at rest, in the center of the nucleus, and, at same time, in orbit around this point, with the further specification that, when they are moving, they would do at the speed of light at a distance equal to the nuclear radius.

In this framework, in fact, the right-hand side of eq. (6) would represent, as we already said, twice the kinetic energy of nucleons $\left(2 * \frac{1}{2} M c^{2}=M c^{2}\right)$.

From the celestial mechanics, indeed, we know that the orbit will be as stable as possible whether the gravitational potential energy will be equal to twice the kinetic energy of the planet, like we have seen in see eq. (1d), reported below:

$$
\frac{G M m}{R}=m v^{2}
$$

from which:

$$
v=\sqrt{\frac{G M}{R}}
$$

(where $M$ is the mass of the Sun) which is notoriously the velocity required to have a circular orbit, namely the most stable orbit.

From eq. (6) it is possible to derive the theoretical formula of the speed of light $c$ :

$$
c=\sqrt{\frac{G K M}{2 \pi R^{3}}}
$$

which is not very different from the planetary orbital velocity seen in eq. (11).

Furthermore in a recent research [6] it has been experimentally shown that the missing momentum of a knockout proton, in some collisions, can be up to $1,000 \mathrm{Mev} / \mathrm{c}$, in contrast with the previous experiments, from which the value of the missing momentum turned out to be 250 $\mathrm{Mev} / \mathrm{c}$.

The value of $1,000 \mathrm{Mev} / \mathrm{c}$ is very high and could be well-justified by assuming that the nucleons move within the nucleus at the speed of light, or at a speed which is approaching it. 


\subsection{Relative facts and absolute facts}

In the reference [7] the authors distinguish relative facts from stable facts, and conclude that the stable facts are only a subset of the more general category of relative facts.

According to this theory, called relational quantum mechanics (RMQ), relative facts are even those concerning the particles that are in two superimposed states, or even the particles that are demonstrated to be ubiquitous, which instead are stable according to quantum mechanics because they are ubiquitous as ubiquitous the decoherence is.

In essence, according to RQM, "Schrödinger's cat has no reason to feel superimposed", because this situation is similar as the man in the Einstein's elevator, which doesn't feel that the elevator, in which he is located, is moving in the interagalactic space (where the absence of gravity is assumed) with uniform linear accelerated motion, but thinks that the elevator is coming up and that he, together with the lift, is subjected to the gravitational force.

In other terms, no matter what the observer sees, the important thing is what the observed feels, what he perceives.

Consequently, if Schrödinger's cat doesn't feel any change after the measurement, then it means that, to cat, nothing has changed, in the sense that, after the measurement, it feels to be in a single state and doesn't perceive any difference with respect to the superimposition situation in which it was before the measurement.

If nothing has changed, it means that no wave function collapse has occurred.

A logical corollary of this fundamental conclusion is that a fact is absolute when the relationality is not possible, namely when observer and observed coincide.

In particular it is possible to arrive at the conclusion that no wave function collapse occurs even by assuming that equation (5) expresses the potential energy of self-orbiting particles (nucleons).

In this framework, in fact, we have assumed that the nucleons revolve around themselves, but this means that the nucleons are observers and observed at same time.

In particular, the orbiting nucleons are revolving particles with respect to their central alter ego, but these latter are not different and separated particles from the orbiting ones: are the nucleons themselves.

Analogously, the central nucleons are at rest with respect to their orbiting alter-ego, but these latter are not different and separated particles from the central ones: are the nucleons themselves.

We can conclude, hence, that the nucleus constitutes a self-interacting system, meaning that the nucleons are observers and observed at same time, and, in this case, the relationality is not possible anymore. 
In fact, claiming that every system is always relative to another one, and consequently that it cannot ever be absolute, holds until observer and observed are different and separated objects or systems, but obviously does not apply when they coincide.

In this particular case, we deal with a system (more precisely a self-interacting system) originating only absolute facts, because the relationality, as necessary requisite for a fact to be relative, lacks.

If the nucleus constitutes a self-interacting system originating only absolute facts, then it means that its wave function cannot collapse, because absolute facts, by definition, cannot collapse, and this is the reason why we are able to see the proofs of this superimposition within the nucleus, as we'll see later.

Finding the evidences of superimposed states is fundamental to demonstrate that this phenomenon truly occurs even before the measurement.

In other words, are we really sure that two entangled photons or electrons are superimposed before measurement?

The question arises because, when we measure (namely observe) one photon entangled to another photon, both of them are never found superimposed, in the sense that the entangled photons manifest themselves in only one state (for instance showing only the spin "up" or only the spin "down"), even if opposed with respect to each other, but never in two states simultaneously.

But the fact that there is the absolute certainty that, when we measure a photon, the nonobserved entangled photon has the opposite spin with respect to the observed photon doesn't necessary mean that the two photons were superimposed before measurement, and that, due to the measurement, they have collapsed in only one status, because we can also reasonably argue that the two photons were moving in that strange, entangled way, even before the measurement, meaning that they were moving in such a way to have in every instant an opposite spin with respect to each other, namely changing their spin continuously and specularly, instant by instant, hence it's obvious that they always show opposite spin after measurement.

Moreover, in order to have the absolute certainty that the two photons were superimposed before the measurement we should observe them in this superimposed state.

Well, in this regard we can say that the nucleons represent a case in which this is possible.

Indeed it has been shown that the nuclear size is bigger than that resulting from the electron scattering experiments.

In particular it has been demonstrated, see reference [8], that a beam of incident particles hitting a target nucleus is both diffracted and absorbed, and, when the absorption is maximum, 
the scattering cross section and the absorption cross section are identical, so that the total cross section, given by the sum between the two cross sections, is twice the scattering cross section.

In particular the particles beam is 50\% diffracted and 50\% absorbed, meaning that the nuclear dimension is twice that detected in the scattering experiments, and that the innermost part of the nucleus is positively charged, whereas the outermost part is neutral.

In fact in the reference [9] it's shown that, in heavy atoms, the absorption of the incident alpha-particles by a target nucleus becomes important at a distance equal to twice the nuclear radius, in agreement with our theory.

The same conclusion are achieved in the references [10] and [11] with regard to the antiprotons beam (negatively charged), whose absorption by the target nucleus is significant even at distances as large as twice the nuclear radius.

This can be well-explained, we repeat, by assuming that the nucleons be self-orbiting particles which are globally charged in while they are at rest and, at same time, electrically neutral in while they are in orbit.

In fact, if we assume that the nucleus be shared into two parts, a half in the center of the nucleus and a half in orbit, then it would follow that the innermost part of the nucleus should be reached by a reduced beam with respect to the originary beam hitting the outermost absorptive part of the nucleus, and in this case the diffracted part of the beam should be smaller than the absorbed part of the beam, but this is not what it happens, because, we repeat, the two part of the beam (that diffracted and that absorbed) are identical, and this can only be (philosophically) explained by arguing that the nucleus not share itself into two parts, but be in a double superimposed state at the same time, namely (i) simultaneously at rest (in the center) and in movement (in orbit), and (ii) simultaneously charged (when at rest) and neutral (when in orbit), and both these superimposed conditions emerge from the mathematical description of the gravitoelectrostatic force contained in eq. (3), $F_{g e s}=\frac{G K M^{2}}{2 \pi R^{4}}$ : the first from the presence of the square of the nuclear mass and from the fact that this force acts as a centripetal force; the second by the simultaneous presence of both the proportionality constants ( $G$ and $K$ ).

Obviously, all these considerations hold under the assumption that the electrostatic repulsion among the central protons be neutralized by neutrons, and that among these central nucleons the gravitoelectrostatic force be only attractive.

But in order to justify the experiments described in the mentioned references [8 - 11] in the light of the gravitoelectrostatic force and gravitoelectric energy proposed in this paper, it's necessary to modify eq. (3) as follows:

$$
F_{g e s}=\frac{4 G K M^{2}}{\pi R^{4}}
$$


Then, eq. (3a) becomes:

$$
\begin{gathered}
U_{g e}=-F_{g e s} * R \\
U_{g e}=-\frac{4 G K M^{2}}{\pi R^{4}} * R \\
U_{g e}=-\frac{4 G K M^{2}}{\pi R^{3}}
\end{gathered}
$$

So eq. (6) becomes:

$$
\frac{4 G K M^{2}}{\pi R^{3}}=M c^{2}
$$

In this way we obtain a value of nuclear radius $\left(R=\sqrt[3]{\frac{4 G K M}{\pi c^{2}}}\right)$ which is exactly equal to twice the radius observed in the electron scattering experiment, and therefore we manage to explain the true, total size of nucleus resulting from both the electron scattering phenomenon and the absorption phenomenon described in the references [8 - 11], provided that we assume that the orbiting nucleons be electrically neutral and that, we repeat, the electrostatic repulsive force among the central protons be neutralized by neutrons.

In order to check whether eq. (12) expresses a force, we have to verify whether in Nature there exists any other case in which there emerges the modification of the gravitational force and potential energy the same way as that done in eq. (13), namely by multiplying the numerator by 4.

For this purpose we can consider the black hole Sagittarius $A^{*}$ existing in the center of Milky way.

We can suppose that the black hole be made up of self-orbiting particles, assumed to be protons, and for this reason we can define it as proton star, whose peculiarity would be, differently from the atomic nucleus, that the charged protons would orbit around their central and neutral alter-egos, whereas in the nucleus, on the contrary, the central core is charged, and the neutral orbiting alter-ego of nucleons are outermost.

Coherently with our assumption that the central alter-ego of protons present in the black hole are neutral, we can suppose that they produce a strengthened gravitational force acting as centripetal force, which constrains the orbiting charged particles, that are moving at the speed of light by their own nature, to revolve around their corresponding central alter-ego.

If this were true, then the gravitational force would become 4 times stronger:

$$
F_{g S}=\frac{4 G M^{2}}{R^{2}}
$$

where $F_{g s}$ is the strengthened gravitational force operating in the black hole, defined like this in order to take into account the quadruplication of its magnitude.

From eq. (1) we can derive the strengthened potential energy $U_{s}$ of the proton star: 


$$
\begin{gathered}
U_{s}=-F_{g s} * R \\
U_{s}=-\frac{4 G M^{2}}{R^{2}} * R \\
U_{s}=-\frac{4 G M^{2}}{R}
\end{gathered}
$$

Now let us apply eq. (2), reported below multiplying both sides by 2 :

$$
-\langle\phi\rangle=2 *\left\langle E_{K}\right\rangle
$$

By supposing that the self-orbiting particles of black hole moves at the speed of light, then the above equation becomes:

$$
\frac{4 G M^{2}}{R}=M c^{2}
$$

Let's replace in the above equation the values of $G, M, R$ and $c$, where $M$ is the mass of Sagittarius $A^{*}$ which, according to [12], is equal to $3.72 * 10^{6}$ solar masses, and $R$ is its radius which, according to [13], is $R \sim 21,979,806,582 \mathrm{~m}$.

$$
\begin{gathered}
\frac{4 *\left(6.6743 * 10^{-11}\right) *\left(3.72 * 10^{6} * 1.9891 * 10^{30}\right)^{2}}{21,979,806,582}=\left(3.72 * 10^{6} * 1.9891 * 10^{30}\right) *(299,792,458)^{2} \\
6.6502 * 10^{53} \text { joule }=6.6502 * 10^{53} \text { joule }
\end{gathered}
$$

It's worth remarking that in other researches [14] it has been demonstrated that the trajectories of particles and of photons within the black hole are compatible with the existence within this entity of self-interacting particles, named gravitational solitons G-Lump.

Therefore we can conclude that the particular forms of potential energies expressed in eq. (13) and in eq. (16), respectively for the atomic nucleus and for the proton star (black hole) — which manifest themselves with the strange presence of 4 in the numerator - seem to be the manifestation of a general principle, inferred inductively, which applies to the self-orbiting systems in which the orbiting part of the system moves at the speed of light.

Therefore we have to conclude, as regards the gravitoelectric potential energy of the nucleus, that the correct equation in not eq. (5), but is eq. (13), reported below:

$$
U_{g e}=-\frac{4 G K M^{2}}{\pi R^{3}}
$$

with the consequence that the correct equation operating in the nucleus is (14), reported again here:

$$
\frac{4 G K M^{2}}{\pi R^{3}}=M c^{2}
$$

with $R$ equal to twice the nuclear radius detected in the electron scattering experiments $(R=2 * 1.21151 * \sqrt[3]{A} \mathrm{fm})$

So now let us compare equations (1d), (14) and (17):

- As concerns planetary orbit [eq. (1d)]: $\frac{G M m}{R}=m v^{2}$ 
- As regards nucleonic orbit [eq. (14)]: $\frac{4 G K M^{2}}{\pi R^{3}}=M c^{2}$

- As regards black hole [eq. (17)]: $\frac{4 G M^{2}}{R}=M c^{2}$

The strong resemblance among these three equations, more than that between eq. (1d) and eq. (6) seen in chapter (4.1), is the evidence of the existence of the natural law expressed in eq. (2), which for clarity is again reported below multiplying both sides by 2 :

$$
-\langle\phi\rangle=2 *\left\langle E_{K}\right\rangle
$$

This definitely confirms what we have already said in chapter 4.1 , namely that the inductive method, rather than the deductive one, represents the best way of approaching and studying gravity and electricity, as well as every natural phenomenon, which have to be accepted as they are, even though they appear strange, weird and not characterized by a precise logic [see all their mysterious aspects described above, namely the inverse square law applicable both to the gravitational and to the electrostatic force $\left(\frac{1}{R^{2}}\right)$, the strange presence of the term $\frac{4}{\pi R^{4}}$ rather than $\frac{1}{R^{2}}$ in the formula of the nuclear gravitoelectrostatic force, the odd presence of number 4 in the formula of the gravitational force operating in the black hole, as well as the aforesaid law contained in eq. (2)].

But, returning to the issue of relative and absolute facts addressed at the beginning of this chapter, at this point a question arises: why can we detect only superimposed states concerning nucleons and not even those concerning photons or, in general, entangled particles?

This question has two possible answers.

The first is to think that the wave function of nucleons, as we have already said, cannot collapse because it involves objects which originate only absolute facts.

The second is to think that the wave function doesn't physically exist, in the sense that it is only a mathematical artifice, with the consequence that the superimposed states which are not detected, but only supposed, have to be considered inexistent until they are experimentally demonstrated.

After all, "entangled" doesn't mean superimposed, but just means "united", "linked" to each other, in the sense that, by measuring only one particle, also the other is immediately affected.

As regards the feature of particles' ubiquity, which manifests itself in the double slits experiment, again it doesn't mean that these particles are superimposed, because being everywhere doesn't mean being simultaneously in two superimposed, opposed states.

Being superimposed means being in two contrary states in the same instant, namely two states which contradict one another, for instance at rest and in movement, charged and neutral, dead and alive, but if a particle moves towards two slits, and passes simultaneously in these slits, it 
doesn't mean that the particle was superimposed, but only that, in while it was moving towards the slits, it was not concentrated only in one point, but was everywhere, yet this is a different situation from the superimposition paradox, and can be also explained by resorting to the pilot wave concept of De Broglie-Bohm.

In this way it is also possible to explain the spherical shape of the nucleus, resulting by the experiments, very likely caused by the pilot wave associated to the orbiting nucleons, which revolve in circular orbit around the center of the nucleus, which would be the center of the resulting sphere.

Anyway the aim of the present paper is seeking to give a response only to the superimposition paradox in microscopic mechanics, and how to understand when it occurs, so we do not go here in the details of the debate concerning the possible interpretations of double slits experiment, which, we repeat, denotes weirdness, not paradoxicalness.

The only thing that we can say in concluding this study is that considering the nucleons as objects originating absolute facts can represent a useful tool to conceptually motivate not only the fact that they do remain superimposed even after the measurement as well as to elucidate the experiments reported in reference [8 - 11], but even to justify some other absolute facts.

In particular, if we accept the existence of self-interacting systems, then we should accept even that the facts they produce can't be other than absolute, for instance the constancy of the speed of light, which is independent from any observer.

The endorsement of the idea that the photons can only produce absolute facts could be supported by arguing that they are in a certain way related to protons, in particular if we think about the possibility that their mass could be equal to the proton mass squared.

In this regards, in the references [15] and [16] it has been remarked that the value of the theoretical mass of the photon is $<2 * 10^{-54} \mathrm{~kg}$, which is not very far from the value of the proton mass squared:

$$
\left(1.6726 * 10^{-27}\right)^{2}=2.7975 * 10^{-54} \mathrm{~kg}
$$

Recently [17] a photon mass of the order of $10^{-54} \mathrm{~kg}$ has been proposed as a possible explanation of the discrepancy between luminosity and red shift distances from some astrophysical sources, like Type Ia supernovae.

Obviously the photon mass will be measured in Kilogram (Kg), not in Kilogram squared $\left(\mathrm{Kg}^{2}\right)$, because the square of the mass is only a mathematical way to express a numerical value, not a substantial element from which to infer the abstract validity or invalidity of the inference.

In other words, applying the empirical inductive method as we have suggested above, we can assert that, if Nature has imposed that the photon must have a mass which is numerically equal to 
the proton mass squared, like it seem to be shown in mentioned references [15 - 17], no abstract human rules can preclude this.

Induction, indeed, implies intuition, followed by the numerical confirmation, and this intuition leads us to hypothesize that the proton, under some conditions specified in chapter 5 , can decay in a photon, with an associated huge release of energy.

In the General Scholium to Book 3 of Isaac Newton's Philosophiae Naturalis Principia Mathematica, third edition, the author said:

"Thus far I have explained the phenomena of the heavens and of our sea by the force of gravity, but I have not yet assigned a cause to gravity. Indeed, this force arises from some cause that penetrates as far as the centers of the Sun and planets without any diminution of its power to act, and that acts not in proportion to the quantity of the surfaces of the particles on which it acts (as mechanical causes are wont to do) but in proportion to the quantity of solid matter, and whose action is extended everywhere to immense distances, always decreasing as the squares of the distances. Gravity toward the sun is compounded of the gravities toward the individual particles of the sun, and at increasing distances from the sun decreases exactly as the squares of the distances as far as the orbit of Saturn, as is manifest from the fact that the aphelia of the planets are at rest, and even as far as the farthest aphelia of the comets, provided that those aphelia are at rest. I have not as yet been able to deduce from phenomena the reason for these properties of gravity, and I do not "feign" hypotheses. For whatever is not deduced from the phenomena must be called a hypothesis; and hypotheses, whether metaphysical or physical, or based on occult qualities, or mechanical, have no place in experimental philosophy.

In this experimental philosophy, propositions are deduced from the phenomena and are made general by induction. The impenetrability, mobility, and impetus of bodies, and the laws of motion and the law of gravity have been found by this method. And it is enough that gravity really exists and acts according to the laws that we have set forth and is sufficient to explain all the motions of the heavenly bodies and of our sea".

The inductive method should guide every natural science, and this work is completely inspired by this modus operandi: the nuclear gravitoelectrostatic force $\left(F_{g e s}=\frac{4 G K M^{2}}{\pi R^{4}}\right)$ and the particular gravitational force operating in black hole $\left(F_{g S}=\frac{4 G M^{2}}{R^{2}}\right)$ have been uncovered by means of the aforesaid method, by supposing the equivalence between the aforesaid forces and the centripetal force (like we will clarify in the chapter 4.6), analogously to the way by which sir Isaac Newton discovered and demonstrated that the force of gravity, exerted by the Sun upon the planets, acts as a centripetal force. 


\subsection{The exact value of the gravitoelectrostatic force}

At this point a question arises.

Which is the correct value of the gravitoelectrostatic force?

That expressed by eq. (3) or that contained in eq. (12)?

The answer is without doubt the force given by eq. (12), not only because it allows to derive the total nuclear radius $R=\sqrt[3]{\frac{4 G K M}{\pi c^{2}}}$, which is in agreement with the results of the nuclear reactions experiments that provide evidences of the fact that the nuclear size is twice that detected in the electron scattering experiments, but also because its value is $\sim 10^{38}$ times stronger than the value of the force of gravity at the same range, which is notoriously the same ratio existing between the strong nuclear force and the gravitational force, whereas the gravitoelectrostatic force expressed by eq. (3) is "only" $10^{37}$ times stronger.

In fact the value of the gravitoelectrostatic force, as expressed in eq. (3), for bromum atom, is:

$$
F_{g e s}=\frac{G K M^{2}}{2 \pi R^{4}}
$$

with $R=1.21151 * \sqrt[3]{79} \mathrm{fm}=5.1983 \mathrm{fm}$

$$
\begin{gathered}
F_{\text {ges }}=\frac{\left(6.6743 * 10^{-11}\right) *\left(8.9875 * 10^{9}\right) *\left\{[(35 * 1.6726)+(44 * 1.6749)] * 10^{-27}\right\}^{2}}{2 * 3.1415 *\left(5.1983 * 10^{-15}\right)^{4}} \mathrm{~N} \\
F_{\text {ges }}=2,286,312 \mathrm{~N}
\end{gathered}
$$

Let us calculate, now, the value of the force of gravity that the nucleus produces upon itself at the range of $R=5.1983 \mathrm{fm}$ :

$$
\begin{gathered}
F_{g}=\frac{G M^{2}}{R^{2}} \\
F_{g}=\frac{\left(6.6743 * 10^{-11}\right) *\left\{[(35 * 1.6726)+(44 * 1.6749)] * 10^{-27}\right\}^{2}}{\left(5.1983 * 10^{-15}\right)^{2}} \mathrm{~N} \\
F_{g}=4.31 * 10^{-32} \mathrm{~N} \\
\frac{F_{g e s}}{F_{g}}=\frac{2,286,312 \mathrm{~N}}{4.31 * 10^{-32} \mathrm{~N}} \\
\frac{F_{g e s}}{F_{g}}=5.29 * 10^{37}
\end{gathered}
$$

Let us now compare the gravitoelectrostatic force, as expressed in eq. (12), and the force of gravity at same range, always in the bromum atom:

$$
F_{g e s}=\frac{4 G K M^{2}}{\pi R^{4}}
$$

with $R=2 * 1.21151 * \sqrt[3]{79} \mathrm{fm}=2 * 5.1983 \mathrm{fm}$

$$
F_{\text {ges }}=\frac{4 *\left(6.6743 * 10^{-11}\right) *\left(8.9875 * 10^{9}\right) *\left\{[(35 * 1.6726)+(44 * 1.6749)] * 10^{-27}\right\}^{2}}{3.1415 *\left(2 * 5.1983 * 10^{-15}\right)^{4}} \mathrm{~N}
$$




$$
F_{\text {ges }}=1,143,156 \mathrm{~N}
$$

Let us calculate the force of gravity that the nucleus produces upon itself at the range of $R=2 * 5.1983 \mathrm{fm}:$

$$
\begin{gathered}
F_{g}=\frac{G M^{2}}{R^{2}} \\
F_{g}=\frac{\left(6.6743 * 10^{-11}\right) *\left\{[(35 * 1.6726)+(44 * 1.6749)] * 10^{-27}\right\}^{2}}{\left(2 * 5.1983 * 10^{-15}\right)^{2}} \mathrm{~N} \\
F_{g}=1.07 * 10^{-32} \mathrm{~N} \\
\frac{F_{g e s}}{F_{g}}=\frac{1,143,156 \mathrm{~N}}{1.07 * 10^{-32} \mathrm{~N}} \\
\frac{F_{g e s}}{F_{g}}=1.05 * 10^{38}
\end{gathered}
$$

This confirms that eq. (12) expresses the correct value of the gravitoelectrostatic force acting within the atomic nucleus, by being $\sim 10^{38}$ times stronger than the force of gravity at same range, in agreement with the experimentations.

Consequently, by knowing that the Coulomb constant is $K=\frac{1}{4 \pi \varepsilon_{0}}$ where $\varepsilon_{0}$ is the dielectric constant in vacuum $\left(\varepsilon_{0}=8.8541 * 10^{-12} \frac{C^{2}}{N * m^{2}}\right)$, the gravitoelectric force expressed in eq. (12) becomes:

$$
\begin{gathered}
F_{g e s}=\frac{4 G M^{2}}{4 \pi \varepsilon_{0} \pi R^{4}} \\
F_{g e s}=\frac{G M^{2}}{\pi^{2} \varepsilon_{0} R^{4}}
\end{gathered}
$$

\subsection{The self-interacting systems and the second principle of dynamics}

Let us report below eq. (14):

$$
\frac{4 G K M^{2}}{\pi R^{3}}=M c^{2}
$$

Let's divide both sides by $R$, intended, we repeat, as twice the nuclear radius detected in the electron scattering experiments $(R=2 * 1.21151 * \sqrt[3]{A} \mathrm{fm})$ :

$$
\frac{4 G K M^{2}}{\pi R^{4}}=\frac{M c^{2}}{R}
$$

The right-hand side represents the centripetal force acting upon nucleons, and this confirms that eq. (12), $F_{g e s}=\frac{4 G K M^{2}}{\pi R^{4}}$, expresses a force, by being equal to another force, the centripetal one.

Equation (19) is also important because it affects the second principle of dynamic. 
In fact, by the gravitoelectrostatic force $F_{g e s}$ being expressed as $\frac{4 G K M M}{\pi R^{4}}$, it follows that the gravitoelectrostatic acceleration $g_{e s}$ is $\frac{4 G K M}{\pi R^{4}}$, and this means that:

$$
F_{g e s}=M * g_{e s}
$$

Therefore, in the self-interacting gravitoelectric systems, such as the atomic nucleus, the second principle of dynamic - which, if applied to the gravitational force, sounds $F=m * g$, where $g$ is the gravitational acceleration and $m$ is the test mass — undergoes the following exception:

$$
F=M * g
$$

with $g$ equal to $\frac{4 G K M}{\pi R^{4}}$

Incidentally, the same reasoning applies to black holes.

Let us report below eq. (17), which above we have seen to be applicable to black hole:

$$
\begin{aligned}
& \frac{4 G M^{2}}{R}=M c^{2} \\
& \frac{4 G M^{2}}{R^{2}}=\frac{M c^{2}}{R}
\end{aligned}
$$

The right-hand side of eq. (20) represents the centripetal force of particles orbiting within the black hole, and this confirms that eq. (15) - which we have defined above as the strengthened gravitational force $\left(F_{g s}=\frac{4 G M^{2}}{R^{2}}\right)$ - expresses a force, by being exactly equal to another force, the centripetal one.

Equation (20) is also relevant because it affects, analogously to eq. (19), the second principle of dynamic $(F=m * g)$.

In fact, by the strengthened gravitational force $F_{g s}$ being equal to $\frac{4 G M M}{R^{2}}$, it follows that the strengthened gravitational acceleration $g_{S}$ is equal to $\frac{4 G M}{R^{2}}$, and this means that:

$$
F_{g s}=M * g_{s}
$$

Therefore, in the self-interacting gravitational systems, such as the black hole, the second principle of dynamic - which states that $F=m * g$, where $g$ is the gravitational acceleration and $m$ is the test mass - undergoes the following exception:

$$
F=M * g
$$

with $g$ equal to $\frac{4 G M}{R^{2}}$

\subsection{The nucleus as an harmonic oscillator}

From eq. (14) it is possible to demonstrate that the atomic nucleus is an harmonic oscillator.

Let us report eq. (14) below: 


$$
\frac{4 G K M^{2}}{\pi R^{3}}=M c^{2}
$$

Let us divide both sides by $M R$ :

$$
\frac{4 G K M}{\pi R^{4}}=\frac{c^{2}}{R}
$$

The right-hand side of the above equation is the centripetal acceleration of nucleons, and the left-hand side is their gravitoelectrostatic acceleration $g_{e s}$ (see previous chapter).

Let us replace the velocity of light $c$ with $\omega * R$ (where $\omega$ is the angular velocity of nucleons), then the eq. (21) becomes:

$$
\begin{aligned}
& g_{e s}=\frac{\omega^{2} * R^{2}}{R} \\
& g_{e s}=\omega^{2} * R
\end{aligned}
$$

which is the equation of a particular harmonic oscillator in which the velocity of the body is constant, and, in such a situation, the radius $R$, which represents the maximum oscillation, can be taken as the projection on the $x$ axis $\left(a=\omega^{2} * x\right)$.

Incindentally, the same reasoning holds even for the black hole. In particular by using eq. (17) we have:

$$
\begin{aligned}
\frac{4 G M^{2}}{R} & =M c^{2} \\
\frac{4 G M}{R^{2}} & =\frac{c^{2}}{R}
\end{aligned}
$$

The right-hand side of the above equation is the centripetal acceleration of particles orbiting within the black hole, and the left-hand side is their strengthened gravitational acceleration $g_{s}$ (see previous chapter).

Let us replace the velocity of light $c$ with $\omega * R$ (where $\omega$ is the angular velocity of orbiting particles), then eq. (22) becomes:

$$
\begin{aligned}
& g_{s}=\frac{\omega^{2} * R^{2}}{R} \\
& g_{s}=\omega^{2} * R
\end{aligned}
$$

(with $R$ equal to $x$ ) which is the proof of the existence of the same particular harmonic oscillator as that previously seen for the atomic nucleus.

\subsection{Physics as experimental philosophy}

The physical law expressed in eq. (2), inferred by induction from the comparison of three specific rules applicable in three particular cases (planetary orbits, nucleonic orbit and selforbiting particles present within the black hole), is certainly very simple.

In the third edition of Principia, Newton sets forth the rules of reasoning in philosophy, and the third rule asserts: 
"The qualities of bodies, which admit neither intension nor remission of degrees, and which are found to belong to all bodies within the reach of our experiments, are to be esteemed the universal qualities of all bodies whatsoever."

In the explanation of the aforesaid rule, the English scientist states:

"We are certainly not to relinquish the evidence of experiments for the sake of dreams and vain fictions of our own devising; nor are we to recede from the analogy of Nature, which uses to be simple, and always consonant to itself".

To Newton, hence, Nature uses to be simple, and always in agreement with itself, namely treating analogous situations in the same way.

Another paramount lesson contained in the Newton's above statement, is that we have not to recede from searching for the analogy of natural phenomena.

Analogy is a fundamental tool to reveal the causes hidden behind the effects and to discover the universal laws of Nature, and in the present study we have sought to follow this method, by finding an analogy between the gravitoelectrostatic force and the centripetal force, analogously to the gravitational force, considered by Newton as a centripetal force acting on the planets of the Solar System.

After all, the use of analogy in physics is nothing else that the consequence of the inductive method, by the induction being essentially relied on empirical results, and in this sense Newton defines physics as "experimental philosophy".

\section{Practical implications of our theory: proton decay?}

At this point a question arises: which could be the consequences of the equality between the gravitoelectric potential energy of nucleons and their kinetic energy?

In macroscopic systems, like gravitational ones, this equivalence implies the fact that the orbit becomes hyperbolic, so the orbital dynamic equilibrium breaks.

By we having demonstrated that the atomic nucleus is subjected to the same rules of macroscopic gravitational system, in particular to the law contained in eq. (2), consequently we are allowed to impose the equivalence between the gravitoelectric potential energy of nucleons and their kinetic energy as a condition to get the breaking of the nucleonic orbital dynamic balance, so eq. (14) becomes:

$$
\begin{aligned}
& \frac{4 G K M^{2}}{\pi R^{3}}=\frac{1}{2} M c^{2} \\
& R=\sqrt[3]{\frac{8 G K M}{\pi c^{2}}}
\end{aligned}
$$


The above equation expresses the extent of the nuclear radius at which the nucleus should lose its identity as self-interacting system, since its constituent elements (nucleons) should become free from their corresponding alter-ego, and this should result in the release of a huge amount of energy, equal to $\frac{1}{2}\left(M_{p} * Z * c^{2}\right)$, where $M_{p}$ is the single proton mass, without any remainder of radioactive isotopes, because all the protons $(Z)$ should turn, if our theory is correct, into energy, in form of light (as a consequence of the proton decay: see the ending of chapter 4.4), kinetic energy and, above all, heat.

As regards neutrons, they should undergo beta-decay, and the protons resulting from this decay should in turn decay in photons with the simultaneous release of the same energy seen above, with the consequence that the total energy released because of the equivalence between the potential energy and the kinetic energy of total nucleons $(A)$ should amount to: $\frac{1}{2}\left(M_{p} * A * c^{2}\right)$.

\section{Conclusion}

In the present study we have shown that the corpuscular aspect of nucleons can be mathematically described, with regard to their dynamics, by means of the Newtonian mechanics, without prejudice to quantum mechanics with respect to its capability to describe their behavior as waves.

In particular, by assuming that the nucleus be a self-interacting system, meaning that nucleons be intended as particles revolving around themselves at the speed of light, by virtue of the gravitoelectrostatic force acting as centripetal force, having this magnitude $F_{g e s}=\frac{G M^{2}}{\pi^{2} \varepsilon_{0} R^{4}}$ (which is $10^{38}$ times stronger than the force of gravity at same range, where $\varepsilon_{0}$ is the dielectric constant in vacuum and $R$ is twice the nuclear radius observed in the electron scattering experiments), and measured in $\frac{\mathrm{N}^{2}}{\mathrm{C}^{2}}$, it has been possible to demonstrate that gravity and electricity merge together within the nucleus.

The necessary premise to accept the gravitoelectrostatic force as a fundamental force of Nature is the acceptance of the equivalence $\frac{\mathrm{N}^{2}}{\mathrm{C}^{2}} \equiv \mathrm{N}$, in turn presupposing the equivalence $\mathrm{C} \equiv \sqrt{\mathrm{N}}$, which makes the International System of Units coherent and absolute without the need of a specific unit of measurement for the electric quantities, analogously to the CGS-ESU system.

The non-perturbative approach proposed in this work presents the advantage of being mathematically very simple - because to assume that the nucleus orbits around itself excludes 
the applicative difficulties of the two bodies problem, reducing the issue to the one body problem - yet, since the numerical value of the gravitoelectric potential energy of nucleons, found in our research, is exactly equal to the numerical value of $M c^{2}$, then one could conclude, by induction, that in the atomic nucleus a typical law of the macroscopic world be operating, namely the fact that the opposite of the gravitational potential energy of the orbiting body $(-U)$ has to be equal to twice its kinetic energy in order to obtain a stable orbit, and, in this case, one should admit that the expression $M c^{2}$ does not denote the total mass-energy of nucleons, like instead the special theory of relativity states, but does express twice the kinetic energy of nucleons, and therefore the foundations of physics regarding the atomic nucleus, as well as those concerning the theory of relativity itself, could be questioned.

A theoretical new form of eco-sustainable exploitation of nuclear energy has been envisaged, under the condition of succeeding in finding a way of enlarging the nuclear radius until to get the equivalence between the gravitoelectric potential energy of nucleons and their kinetic energy, and only the experimentations can show which is the best way of getting it.

\section{Data Accessibility}

Preprint: https://doi.org/10.31219/osf.io/68fbs

\section{Competing Interests}

The author declares no competing interests

\section{References}

[1] Bogdan Povh, Klaus Rith, Christoph Scholz, Frank Zetsche, Martin Lavelle, Particles and Nuclei. Springer, Berlin, Heidelberg; 10.1007/978-3-662-46321-5 (2015)

[2] Nörtershäuser W, et al. Nuclear charge radii of 7, 9, 10Be and the one-neutron halo nucleus 11Be. Phys Rev Lett. 102(6):062503; 10.1103/physrevlett.102.062503 (2009)

[3] Richard Feynman (1970). The Feynman Lectures on Physics Vol II. Addison Wesley Longman. pp. 1-3, 1-4. ISBN 978-0-201-02115-8

[4] Purcell, Edward M.; Morin, David J. (2013). Electricity and Magnetism (3rd ed.). New York. Cambridge University Press. pp. 15-16. ISBN 978-1-107-01402-2

[5] Serway, Raymond A.; Vuille, Chris (2014). College Physics, 10th Ed. Cengage Learning. pp. 532-533. ISBN 978-1305142824

[6] Schmidt, A., et al., Probing the core of the strong nuclear interaction. Nature 578, 540-544; $\underline{10.1038 / \mathrm{s} 41586-020-2021-6}$ (2020) 
[7] Di Biagio, A., Rovelli, C. Stable Facts, Relative Facts. Found Phys 51, 30. 10.1007/s10701$\underline{021-00429-\mathrm{w}}(2021)$

[8] C.A. Bertulani, Nuclear reactions, Wiley Encyclopedia of Physics. ISBN-13: 978-3-52740691-3. Wiley-VCH, Berlin (2009). Preprint at https://arxiv.org/abs/0908.3275

[9] G. Michaud, L. Scherk, and E. Vogt, Nuclear Optical Model and Wave Properties: Barrier Penetration, Reflection, Absorption, and Resonance. Phys. Rev. C 1, 864; 10.1103/PhysRevC.1.864 (1970);

[10] S. Wycech et al., Antiprotonic Studies of Nuclear Neutron Halos. Phys. Rev. C 54, 281832 10.1103/PhysRevC.54.1832 (1996)

[11] Wycech S., Smolańczuk R., Nuclear Neutron Haloes as Seen by Antiprotons. In: Adam J., Dobeš J., Mach R., Sotona M., Dolejší J. (eds) Mesons and Light Nuclei '95. Few-Body Systems, vol 9. Springer, Vienna; 10.1007/978-3-7091-9453-9_12 (1995)

[12] Ghez, A. M., Salim, et al. Stellar orbits around the galactic center black hole. ApJ 620, 744; $\underline{10.1086 / 427175}(2005)$

[13] Doeleman, S., Weintroub, J., Rogers, A. et al., Event-horizon-scale structure in the supermassive black hole candidate at the Galactic Centre. Nature 455, 78-80; $\underline{10.1038 / \text { nature } 07245}$ (2008)

[14] Irina Dymnikova and Anna Poszwa, Classification and basic properties of circular orbits around regular black holes and solitons with the de Sitter center. Class.Quantum Grav. 36 $105002 ; \underline{10.1088 / 1361-6382 / a b 150 c}$ (2019)

[15] A. Proca, Sur la théorie ondulatoire des électrons positifs et négatifs. J. Phys. Radium 7, 347; 10.1051/jphysrad:0193600708034700 (1936)

[16] Julian Heeck, How Stable is the Photon? Phys. Rev. Lett. 111, 021801; 10.1103/PhysRevLett.111.021801 (2013)

[17] Spallicci, A.D.A.M., Helayël-Neto, J.A., López-Corredoira, M. et al. Cosmology and the massive photon frequency shift in the Standard-Model Extension. Eur. Phys. J. C 81, 4. $\underline{10.1140 / \mathrm{epjc} / \mathrm{s} 10052-020-08703-3}$ (2021) 